\title{
Percutaneous drainage of thoracic wall cold abscess with Malecot's catheter -indications and pitfalls
}

\author{
Bhatnagar Ankur ${ }^{1}$, Mazumdar Gaurango ${ }^{2}$, Pandey Shantanu ${ }^{2}$, Agarwal S. K. ${ }^{2}$, Gupta Devendra ${ }^{3}$ \\ ${ }^{1}$ Department of Plastic Surgery, SGPGIMS, Lucknow, India \\ ${ }^{2}$ Department of Cardiovascular and Thoracic surgery, SGPGIMS, Lucknow, India \\ ${ }^{3}$ Department of Anaesthesia, SGPGIMS, Lucknow, India
}

\section{Email address:}

bhatnagarankur2000@yahoo.com (B. Ankur)

\section{To cite this article:}

Bhatnagar Ankur, Mazumdar Gaurango, Pandey Shantanu, Agarwal S. K., Gupta Devendra. Percutaneous Drainage of Thoracic Wall Cold Abscess with Malecot's Catheter -Indications and Pitfalls. Journal of Surgery. Vol. 2, No. 3, 2014, pp. 50-53.

doi: $10.11648 /$ j.js.20140203.14

\begin{abstract}
Chest wall cold abscess is a rare presentation. Tubercular localization in the thoracic cage is rare and difficult to diagnose, due to multiple clinical presentations with chest wall cold abscess being the commonest. Cold abscess of the chest wall must be treated more aggressively, and meticulous debridement and wide resection including involved bones and cartilages is required followed by coverage with local muscle or musculocutaneous flaps. Tube drainage under adequate ATT cover is a viable treatment option especially in patients not amenable to surgical intervention. Sinus formation after tube drainage reflects inadequate medical treatment. Small bore tubes should be used, which should be removed once radiological and clinical evidence of diseases resolution is obtained. If Malecot's catheter is left for a long time in the cold abscess cavity, it get's retained due to soft tissue in-growth from the collapsing cavity into the catheter tip and wings. The tissue in-growth and fibrosis can lead to complete integration of this foreign body into the tissues, which can only be removed through a surgical procedure. In such cases Malecot's catheter is removed en-masse with a cuff of granulation tissue to ensure complete clearance of the foreign material.
\end{abstract}

Keywords: Cold Abscess, Malecot's Catheter

\section{Introduction}

Tuberculosis (TB) and its management is a major health issue world over. Although safe and effective antitubercular (ATT) chemotherapy is available, emergence of multidrug-resistant (MDR), Extensively drug-resistant (XDR) TB and TB in immune-compromised individuals presents a new challenge for health care individuals. Whereas the lungs are the main target in primary infection, extra-pulmonary tuberculosis has been reported to constitute $15-20 \%$ of all tuberculosis cases [1]

Chest wall TB although rare can cause diagnostic dilemma. Tubercular localization in the thoracic cage is rare and difficult to diagnose, due to multiple clinical presentations with chest wall cold abscess being the commonest [2]. Surgical debridement and ATT alone or in combination has been the preferred mode of treatment, however optimal management especially in immune-compromised individuals or patients with multiple co-morbidities is still controversial [3]. Malecot's cather is a self retaining catheter used for drainage of intra-abdominal collections in particular [4].

\section{Case Presentation}

65 year old male presented to us with history of chronic renal failure on hemodialysis for last 8 months. Five months back he had noticed a large soft tissue mass in the right side chest wall in the periareolar area. Lesion was non tender, fixed to the underlying muscle with abscense of any pulsations. Investigations were consistent with chronic renal failure with serum Creatnine of 6.5. Ulrasonography (USG) showed an extra-pleural abscess cavity deep to the intercostal muscles. X-ray confirmed the cavity with minimal or no rib involvement. A diagnosis of extra-pulmonary TB with cold abscess of the chest wall was made after FNAC of the lesion along with radiography. Due to poor general condition of the patient and deranged 
renal function, an USG guided Malecot's catheter (Fig 1,2) was put into the abscess cavity to drain out pus in the department of radiology. Patient was put on ATT following drainage and then lost to follow-up. He reported to outpatient department five months later. On examination the abscess cavity had collapsed with no tube drainage. However manual removal of the Malecot's catheter was not possible. The catheter was retained in the deeper tissues, and pulling on the catheter caused severe chest pain to the patient.

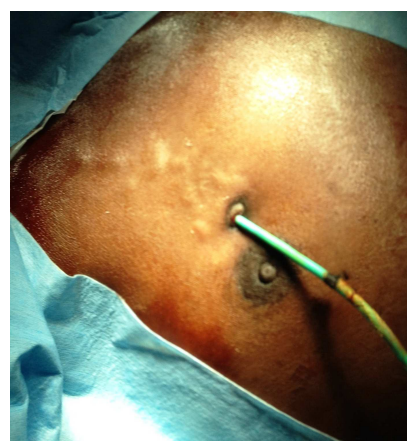

Fig 1. Retained malecot's catheter in chest wall

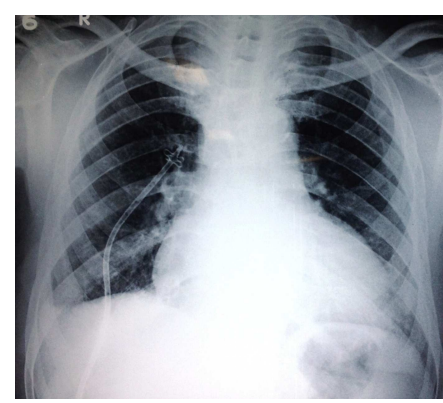

Fig 2. X-Ray showing catheter in situ

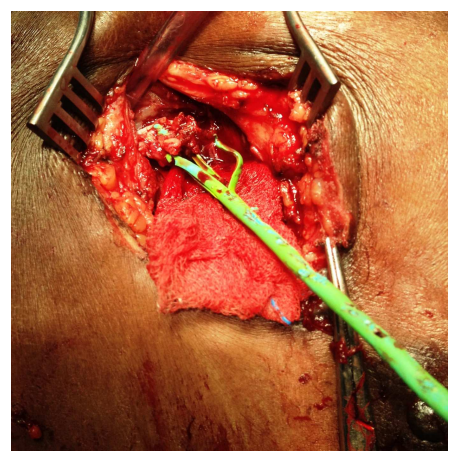

Fig 3. Catheter tip engulfed in granulation tissue

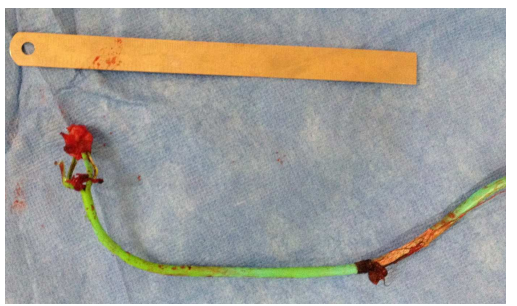

Fig 4. Catheter removed with cuff of granulation tissue

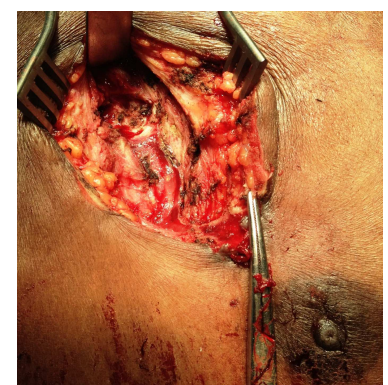

Fig 5. Complete clearance of the catheter

It was then decided to surgically remove the catheter under short general anaesthesia (GA). The catheter insertion site was incised; however in-spite of gentle tugging it was impossible to remove it. Entire catheter tract till the Malecot tip was subsequently laid open. Catheter tip was found completely engulfed by the granulation tissue just superficial to the parietal pleura in the intercostal space [Fig 3]. Catheter tip could not be freed from the granulation tissue. Hence the entire tip along with a cuff of granulation was excised en-mass, taking care not to leave any part of the Malecot tip in the cavity[Fig 4]. Complete clearance was ensured [Fig 5]. Closure was done in layers after ensuring haemostasis. Post operatively, the entire suture line healed well, with absence of an sinus formation.

\section{Discussion}

Although lungs are the main target in primary infection, extra pulmonary tuberculosis constitutes $15-20 \%$ of all tuberculosis cases . Chest wall tuberculosis may occur by means of two mechanisms: (1) haematogenous dissemination associated with activation of a dormant tuberculous foci and (2) direct extension from chest wall lymphadenitis .Development of cold abscess is the hall mark of chest wall TB. It has been postulated that tuberculous bacilli invade the pleural space leading to local or widespread pleuritis; some bacilli are then transported from the pleural space to the parasternal (or posterior intercostal) lymph nodes; which undergo casseous necrosis and rupture. This necrotic and casseous material burrows anteriorly or posteriorly to form a cold abscess in the chest wall[6].

Cold abscess can be confused with chest wall tumour and rib TB. Chest wall tuberculosis usually presents as a painless mass. This palpable mass is frequently cystic or soft, and may fluctuate on physical examination. Local tenderness or erythema signifies secondary infection which is usually absent [7]. Presence of Caseous granulomatous necrosis or Langhans giant cell from biopsy or FNAC specimens, confirms the diagnosis. FNAC, being less invasive is the initial diagnostic procedure however it may not be reliable as the aspirated casseous material may not contain typical giant cells. Hence in cases with strong clinical suspicion surgical biopsy becomes the gold standard [8]. Radiography and CT scan can aid in the 
diagnosis which can be confirmed by tissue aspirates/curettage. MRI is not deemed necessary. USG can elegantly demonstrate the rib destruction/irregularity and the associated abscesses, especially in accessible regions of the chest wall, thus providing a low cost alternative to CT scan. In PET/CT these lesions are seen as areas of increased FDG uptake in active regions of granulomatous inflammation, with cold areas representing necrosed tissue[8].

The treatment of chest wall tuberculosis is controversial. There are some series reporting good results with only ATT while in others, abscesses were not cured and even recurred or progressed despite adequate medical treatment[3]. It is generally regarded that combination of ATT with wide surgical debridement including rib resection, if involved, remains the best treatment option with least recurrence rates [9]. In all such cases the residual cavity post resection is generally not closed to allow adequate drainage via an indwelling catheter left in situ, ranging from a few days to couple of weeks. It is believed that long term catheter insertion may lead to a sinus formation [7].

However in our search of literature, we could not find adequate treatment guidelines for patients with multiple co-morbidities especially who are not fit for surgery, as in our case.

Malecot's is a self retaining catheter with a bulbous tip and multiple wings, both the tip and the wings prevent accidental catheter dislodgement. Percutaneous catheter drainage (PCD) of abscesses adheres to the basic principles of surgical management by providing decompression, evacuation and continuous drainage without dissemination of sepsis. Its safety, simplicity and excellent cure rates in drainage of abdominal abscesses/collections have established it as an alternative to surgical drainage in intra-abdominal collections[10]. However, only limited literature is available, regarding per cutaneous drainage in tuberculous abscesses, with some reports showing good results in ilio-psoas tuberculous abscesses along with ATT coverage[10].All such reports suggest that the catheter should be retained for at-least one month during which time ATT coverage should be given. WHO recommends a standard 6-month regimen, which can be extended up to 9-12 months [11].

In consultation with patient's attendants, who were not willing for any major surgical intervention, it was decided to start ATT and perform USG guided abscess drainage. It was also decided to remove the Malecot's catheter once drainage ceased from the tube and there was radiological evidence of a resolving abscess. However patient was lost to follow-up and persisted with catheter in situ for five months.

Two facts have come out in our case; firstly there was no sinus formation after catheter removal in our patient in-spite of the prolonged catheter insertion. This could be attributed to effective ATT which the patient continued to take at his local place. Secondly the prolonged Malecot's retention brought to light a lesser known complication i.e retained catheter due to soft tissue in-growth from the collapsing cavity into the catheter tip and wings. The tissue in-growth and fibrosis led to complete integration of this foreign body into the tissues, which could only be removed through a surgical procedure. Unlike abdominal collections which are mostly acute in nature, cold abscesses are chronic lesions, having extensive granulation tissue. In abdominal cavity it is possible to retain catheters for a long time without fear of granulation tissue growth, moreover the bulbous tip and large wings prevents accidental dislodgement of the catheter from the abdomen. However we believe that in cold abscess the bulbous tip and large wings act as a nidus for granulation tissue formation, left long the large malecot tip thus get's integrated into the granulation tissue making manual removal almost impossible. Hence, in all such cases of cold abscess small bore catheter should be used which should be removed as soon as the drainage ceases and there is radiological evidence of abscess resolution. Sinus formation after long term catheter use only signifies inadequately controlled tubercular pathology. Once a retained Malecot's is encountered manual pulling should be avoided as this can lead to catheter breakage especially part of the tip and wings, leading to secondary infection and sepsis. Surgery should be meticulous where, complete clearance of the foreign body should be insured, and this can be done by taking out the catheter en-mass with the granulation tissue thus preventing all chances of foreign body retention.

\section{Conclusion}

Chest wall cold abscess is a rare presentation. Cold abscess of the chest wall must be treated more aggressively, and meticulous debridement and wide resection including involved bones and cartilages is required followed by coverage with local muscle or musculocutaneous flaps. Tube drainage under adequate ATT cover is a viable treatment option especially in patients not amenable to surgical intervention. Sinus formation after tube drainage reflects inadequate medical treatment. Small bore tubes should be used, which should be removed once radiological and clinical evidence of diseases resolution is obtained. In cases where Malecot's catheter is retained due to prolonged use surgical removal should be done ensuring complete removal of the catheter. Manual force-full pulling of the draining tube should be avoided to prevent retained foreign body in the collapsed cavity.

\section{References}

[1] Iseman MD. Extrapulmonary tuberculosis in adults. In: Iseman MD, editor. Clinician's guide to tuberculosis. Philadelphia: Lippincott; 2000. p. 145-97

[2] Tuberculosis of rare sites, girdle and flat bones. In: Tuli SM, editor. Tuberculosis of the Skeletal System (Bones, Joints, Spine and Bursal Sheaths). 2nd ed. New Delhi: Jaypee Brothers Medical Publishers (P) Ltd; 2000. p. 155-60. 
[3] Hsu HS, Wang LS, Wu YC, Fahn HJ, Huang MH. Management of primary chest wall tuberculosis. Scand J Thorac Cardiovasc Surg 1995;29:119-23.

[4] Carr, H. A. A short history of the Foley catheter: from handmade instrument to infection-prevention device. J Endourol $2000 ; 14(1): 5-8$

[5] .Ishizu K, Yamaguchi S, Naito K. A case of multiloculated retroperitoneal abscess successfully treated by percutaneous drainage with a Malecot catheter Hinyokika Kiyo. 1999 Feb;45(2):103-5. Japanese

[6] Burke HE. The pathogenesis of certain forms of extrapulmonary tuberculosis: Spontaneous cold abscesses of chest wall and Pott's disease. Am Rev Tuberc 1950;62:48-67

[7] Aghajanzadeh M, Pourrasouli Z, Aghajanzadeh G,
Massahnia S. Surgical Treatment of Chest Wall TuberculosisTanaffos (2010) 9(3), 28-32

[8] Grover et.al. Chest wall tuberculosis - A clinical and imaging experience. Indian $\mathrm{J}$ Radiol Imaging 2011;21(1):28-33

[9] Paik HC, Chung KY, Kang JH, Maeng DH. Surgical treatment of tuberculous cold abscess of the chest wall. Yonsei Med J 2002;43: 309-14

[10] Puri S K, Panicker H, Narang P, Kumar N, Dhall A, Gupta S B. Percutaneous drainage of tuberculous abscesses. Indian J Radiol Imaging 2001;11:13-6

[11] Kuzucua A, Soysala O, Günenb H. The role of surgery in chest wall tuberculosis. Interact Cardiovasc Thorac Surg 2004; 3: 99-103 